of drugs, complementary health products, and medical devices.

In February 2003 a report by senior academic staff at all four of New Zealand's medical schools, "For Health or Profit?," proposed a ban on such advertising and the establishment of an independent advisory service for doctors and consumers. The report persuaded Ms King to review drug advertising standards.

New Zealand and the United States are the only developed countries that permit direct to consumer advertising for prescription drugs. A spokesman for Ms King was tight lipped on the prospect of a ban on such advertising, stating only that the cabinet would finalise the details later this month.

The chief body in New Zealand for the drugs industry, Researched Medicines Industry Association of New Zealand, declined to comment on the basis that the final decision had not been made. However, on its website the association argues that brand name advertising can "increase patient understanding and awareness of health problems, which can influence patients to seek early advice about treatment."

Bob Burton Canberra

\section{Scottish doctors will have to register financial links to drug companies}

Doctors in Scotland are to be required to declare any financial or personal links they have with drug companies on official registers that will be made available for public inspection. The move is part of an agreement between the NHS and the drugs industry on joint working that aims to reduce potential conflicts of interest and improve openness and transparency.

The agreement is the first of its kind in the United Kingdom and may lead to pressure for similar action elsewhere. Joe Collier, editor of the Drug and Therapeutics Bulletin and professor of medicines policy at St
George's Hospital, London, called for registers to be established across the United Kingdom. "In principle this is a wonderful idea, and I would like to see it extended," he said.

The report welcomes joint working between the NHS and the drugs industry and recognises the potential benefit to patients. However, it also acknowledges that the relationship needs to be controlled and recommends the introduction of better monitoring arrangements. NHS boards are instructed to establish registers of interest of all NHS employees' links with drug companies, including share holdings, lecture fees, payments to attend meetings, and research grants. The report also says that offers from drug companies that breach relevant codes of practice must be reported to the relevant NHS organisation and that concerns about the conduct of any company should also be reported.

The agreement was welcomed by Dr Des Spence, Scottish spokesman for the No Free Lunch movement, which campaigns against doctors accepting money, gifts, or hospitality from the industry. "This is a very positive move," he said. "It is a good attempt to try to clarify the relationship between the industry and the profession. The only reservation I have is about how it is going to be policed. There is no real clarity about what should and should not be declared, and it is being left up to NHS trusts to police it, which may lead to variations in the way it is applied across the country."

Professor Collier also emphasised the importance of defining what needs to be declared and the action that will be taken against anyone found not to be making a full declaration. "It is not all that straightforward. There are edges that need to be looked at seriously."

A spokesman for the Department of Health said there were no current plans to introduce a similar system in England. Bryan Christie Edinburgh

A Common Understanding: Guidance on Joint Working between NHSScotland and the Pharmaceutical Industry is available at www.scotland.gov.uk/ publications

\section{Cognitive behaviour therapy affects brain activity differently from antidepressants}

\author{
Sue Mayor London
}

Cognitive behaviour therapy triggers a different pattern of changes in brain activity than that triggered by the antidepressant paroxetine, according to a small pilot study that provides the first scan based evidence that the treatments work in different ways.

The researchers scanned 17 previously untreated patients with unipolar depression before and after a 15-20 session course of outpatient cognitive behaviour therapy. The group's mean score on the 17 item Hamilton depression rating scale, which rates items on a scale from 0 (absent) to 4 (very severe), was $20 \pm 3$ before treatment. The score improved to $6.7 \pm 4$ in the 14 patients who completed cognitive behavioural therapy. Positron emission tomography was used to examine changes in the brain. The tomography showed significant metabolic changes in the patterns of brain activity in the patients who underwent cognitive behaviour therapy, with increased activity in the hippocampus and dorsal cingulate regions and decreases in cortical activity (Archives of General Psychiatry 2003;61:34-41).

The results were compared after the study with an independent group of 13 men with depression (mean Hamilton depression rating scale score 22.4 \pm 3.6 ) who were scanned

before and after six weeks of treatment with the antidepressant paroxetine. Scans showed changes in brain activity in similar regions as those triggered in patients who underwent cognitive behaviour therapy but in the opposite direction, with increases in activity in the prefrontal cortex and decreases in the hippocampal and dorsal cingulate regions, which are located in the medial part of the temporal lobe.

The Canadian researchers said that-like other treatments for depression-cognitive behaviour therapy seems to help by modulating the function of specific sites in the limbic and cortical regions of the brain. However, they proposed that the difference in the direction of the changes in brain activity may reflect specific effects relating to the type of treatment.

One of the researchers, Helen Mayberg, professor of psychiatry at the University of Toronto, said: "Our imaging results suggest that you can correct the depression network along a variety of pathways. Antidepressant drugs change the chemical balance in the brain through effects at very specific target sites. Cognitive behaviour therapy also changes brain activity: it's just tapping into a different component of the same depression circuit board." 